\title{
Potencial atrator de peixes ósseos em recife artificial no litoral norte do estado do Rio de Janeiro, Brasil
}

\author{
Marcelo Paes Gomes ${ }^{1}$ \\ Ronaldo Novelli ${ }^{1}$ \\ Vicente Vieira Faria ${ }^{2}$ \\ Ilana Rosental Zalmon ${ }^{1}$
}

\begin{abstract}
Attractor potential of osteichthyes in artificial reef on the northern coast of Rio de Janeiro State, Brazil. Artificial reefs have become an important and popular resource enhancement technique by concentrating fishes and by increasing natural production of biological resources. In order to increase the necto-benthic colonization potencial, an artificial reef was installed on the northern coast of Rio de Janeiro ( $\left.21^{\circ} 27^{\prime} \mathrm{S}, 41^{\circ} 00^{\prime} \mathrm{W}\right)$, an area with typically low relief bottom. Measuring nearly $1500 \mathrm{~m}^{2}$, the reef consisted of four sets of different materials randomly disposed: concrete pipes $(\mathrm{N}=12)$; tires structures $(\mathrm{N}=20)$; and cement tanks $(\mathrm{N}=7)$ and pre-made blocks $(\mathrm{N}=4)$. In order to determine the artificial reef effects on the teleost community, trammel nets were used for monthly sampling the reef site (RA) and on a control area $(\mathrm{AC})$ with sandy bottom. During the 23-month survey from A pril/96 to March/98, were recorded: a) Chaetodipterus faber (Broussonet, 1782) and Haemulon aurolieatum (Cuvier, 1829) as exclusive species of the RA; b) higher values of species richness and abundance on the RA, at least in 5 of 8 periods; c) increase on the fish abundance on summer months. Correlation analysis indicated that salinity and precipitation were the most significant environmental factors correlated with the temporal fish community variation. This results highlight the importance of rainfall periodicity and the influence of Paraiba do Sul River on the nekton assemblage distribution. It is suggested that the functional role of the artificial reef might be related to higher availability of local shelter and food resources.

KEY WORDS. Artificial reef, osteichthyes, community structure, temporal variation, Northern coast of Rio de Janeiro.
\end{abstract}

Em ambientes aquáticos, o uso de substratos artificiais de pequeno e grande porte tem se mostrado particularmente indicado como subsídio para algumas linhas de pesquisa, tanto com propósitos comerciais para implementar áreas de maricultura e recuperação costeira (SEAMAN et al. 1989), como de estudos sobre fenômenos ecológicos focalizando as interações entre os organismos (JACOBI 1994; ZALMON et al. 1993).

Atualmente, os recifes artificiais apresentam-se como boas alternativas para a maricultura intensiva, para o aumento da produtividade pesqueira e com enorme potencial para a recuperação de áreas costeiras degradadas. O que se observa,

1) Laboratório de Ciências Ambientais, Universidade Estadual do Norte Fluminense. Avenida Alberto Lamego 2000, Horto, 28015-620 Campos dos Goytacazes, Rio de Janeiro, Brasil. E-mail: marcelopgomes@hotmail.com

2) Department of Zoology and Genetics, Iwoa State University. Ames, Iwoa 50011, USA.

Revta bras. Zool. 18 (3): 779 - 792, 2001 
geralmente, em qualquer recife, é um enorme incremento na biomassa de invertebrados bênticos, redução na mortalidade de espécies que dependem de substrato duro para desova, proteção de espécies que se alojam no recife, atração e concentração de espécies pelágicas que se alimentam no próprio e nos arredores do recife (STONE 1986). Porém, seu papel atrator é discutido, pois primeiramente ocorre um deslocamento de biomassa de ambientes naturalmente estabelecidos para artificiais, podendo prejudicar o primeiro (BOHNSACK et al. 1997).

Um dos principais objetivos de programas de incremento de recursos costeiros tem sido a melhoria do ambiente para atrair espécies desejáveis, tanto da fauna quanto da flora. Dentre os problemas mais comuns identificados como responsáveis pela baixa produtividade pesqueira, destacam-se a escassez de substrato apropriado em áreas onde o fundo consiste tipicamente de substrato arenoso e a intensidade na qual a área encontra-se impactada antropicamente, seja por uma sobre-explotação dos recursos pesqueiros (MORENO et al. 1994; BOMBACE et al. 1994), seja pela poluição imposta ao ambiente. Em resposta, incentivos crescentes envolvem a colocação de estruturas no ambiente marinho que, por sua vez, fornecem substrato para a fixação dos organismos, além de aumentar a complexidade de habitats, através da disponibilidade de espaços verticalmente definidos (MOTTET 1986).

Neste estudo, buscou-se avaliar o potencial pesqueiro numa área sob influência de um recife artificial, utilizando como comparação uma área controle sem influência do mesmo.

\section{MATERIAL E MÉTODOS}

\section{Área de estudo}

O recife artificial (RA) foi instalado em março de 1996 na Enseada de Manguinhos (São Francisco do Itabapoana, Rio de Janeiro) a uma distância aproximada de 5 milhas da costa $\left(21^{\circ} 27^{\prime} \mathrm{S}, 41^{\circ} 00^{\prime} \mathrm{W}\right)$, a uma profundidade média de 9 m. Situa-se a cerca de $25 \mathrm{~km}$ ao norte do estuário do rio Paraíba do Sul. A região é incluída por VALENTIN \& MONTEIRO-RIBAS (1993) em uma zona de transição, influenciada ao norte por águas oligotróficas da Corrente do Brasil e ao sul pela ressurgência da Água Central do Atlântico Sul. As águas da região apresentam-se claras nos meses de verão, aumentando a turbidez e turbulência à medida que se aproximam os meses de inverno. $\mathrm{O}$ fundo, plano, é constituído tipicamente de lajes homogêneas, salvo nas proximidades da praia, onde predominam aglomerados de rochas submersas recobertas por macroalgas.

\section{Parâmetros ambientais}

Em campo, foram monitoradas mensalmente no recife artificial as seguintes variáveis abióticas: temperatura $\left({ }^{\circ} \mathrm{C}\right)$, salinidade e transparência da água $(\mathrm{m})$, através de termossalinômetro e disco de Secchi graduado, respectivamente. Dados de vazão do rio Paraíba do Sul e médias do volume de precipitação foram adaptados de SouZA et al. (1998).

\section{O Complexo Recifal}

Conforme Risk (1981) e PEARCE \& CHANG (1982), um recife artificial deve satisfazer dois critérios: a) ser construído de material localmente abundante e 
relativamente barato e b) apresentar, quando pronto, uma ampla variedade de interstícios e cavidades. Uma grande variedade de formatos dos módulos é descrita na literatura, sendo o formato então característico para cada caso, ou seja, não é necessário seguir um padrão morfológico ou morfométrico (JARA \& CÉSPEDES 1994; GROvE et al. 1994; LINDQUIST et al. 1994).

Em nosso estudo, foram utilizados quatro tipos de substratos experimentais: manilhas de concreto $(\mathrm{N}=12)$; módulos de pneus $(\mathrm{N}=20)$ e cimento pré-moldado em forma de tanques $(\mathrm{N}=7)$ e tijolos $(\mathrm{N}=4)$. Os substratos foram aleatoriamente distribuídos e distavam entre si de 3 a $5 \mathrm{~m}$, totalizando uma área de ocupação de cerca de $1500 \mathrm{~m}^{2}$.

\section{Monitoramento do complexo recifal}

Como dispositivo de amostragem da ictiofauna foram utilizadas, mensalmente, durante cerca de $24 \mathrm{~h}$, conjuntos de cinco redes de espera de fundo cada qual medindo $25 \mathrm{~m}$ de comprimento por $3 \mathrm{~m}$ de altura, com malha entre nós adjacentes de $20,30,40,50$ e $60 \mathrm{~mm}$. As redes eram dispostas tanto no recife artificial quanto em área-controle (AC) não protegida. Esta última distava cerca de $800 \mathrm{~m}$ do complexo recifal, visando uma amostragem sem influência do mesmo que, segundo RYU (1995), é evidenciada num raio de aproximadamente 300 metros.

Em laboratório, os peixes capturados eram descongelados, identificados segundo FigueIREDO \& MENEZES (1978, 1980), MENEZES \& FigueIREDO (1980, 1985) e quantificados. Foram obtidos dados de riqueza de espécies e abundância em termos de número de indivíduos e em peso $(\mathrm{kg})$, em balança digital $(0,1 \mathrm{~g}$ de precisão).

\section{Cálculo da captura por unidade de esforço (CPUE)}

A CPUE é definida como a razão entre o número de indivíduos ou peso capturados e o esforço de pesca despendido para tal, sendo que o esforço é igual ao tamanho da rede $\left(375 \mathrm{~m}^{2}\right.$ por conjunto de cinco redes) por número de barcos (1) por tempo de pesca (um dia). Como meio flutuante, utilizou-se uma traineira por coleta. O tempo de pesca foi estimado de acordo com o número de coletas por período. Assim sendo, calculou-se o esforço total e de cada estação do ano, tanto no recife artificial quanto na área controle.

\section{Tratamento e análise dos dados}

Os dados de riqueza de espécies, abundância em número de indivíduos, abundância em peso e CPUE dos peixes capturados no RA e na $\mathrm{AC}$ foram comparados entre si através de ANOVA mono-fatorial $(\mathrm{p}<0,05)$. Os requisitos de normalidade e homocedascitidade (SOKAL \& ROHLF 1981) foram obtidos através da transformação logarítmica $\log (\mathrm{x})$.

\section{Análise de correlação entre parâmetros ambientais e bióticos}

Com o objetivo de complementar a análise dos grupamentos investigados, procurou-se determinar a influência dos diferentes parâmetros ambientais monitorados na distribuição temporal dos peixes capturados no recife artificial e na área controle. 
As variáveis riqueza de espécies, abundância em número de indivíduos (n), abundância em peso $(\mathrm{kg})$ e CPUE ( $\mathrm{n}$ e $\mathrm{kg}$ ) foram selecionadas como atributos estruturais para análise de correlação de Pearson com os parâmetros ambientais. A significância das correlações foi testada através do teste $t$ de Student $(\mathrm{p}<0,05)$ (ZAR 1999). (1993).

Estas análises foram feitas no programa Statsoft Statistica for Windows 4.5

\section{RESULTADOS}

Após uma análise exploratória em que se observou uma ocorrência tipicamente sazonal quanto aos meses de estudo por grande parte das espécies capturadas, optou-se por uma análise das variações temporais em escala estacional, de modo a evidenciar as mudanças estruturais da comunidade de peixes e das variáveis ambientais em questão.

\section{Parâmetros ambientais (Fig. 1)}

Os valores de vazão e precipitação mostraram um padrão semelhante de sazonalidade, com valores superiores no verão/97 e inferiores no inverno/97. Tal padrão era esperado, visto ser o verão uma estação mais chuvosa e o inverno uma estação mais seca. A vazão variou entre $350 \mathrm{~m}^{3} / \mathrm{s}$ no inverno $/ 96$ e $1443 \mathrm{~m}^{3} / \mathrm{s}$ no verão/97, e os valores de precipitação total variaram entre $5,1 \mathrm{~mm}$ no inverno/96 e $103,9 \mathrm{~mm}$ na primavera/97.

Os valores de temperatura da água apresentaram-se inferiores no inverno/96 $\left(22,4^{\circ} \mathrm{C}\right)$ e mais elevados na primavera/ $/ 97\left(25,4^{\circ} \mathrm{C}\right)$.

Os valores de salinidade apresentaram uma flutuação irregular, variando entre 29,8 no verão/97 (período mais chuvoso) e 36,2 no outono/96 (período mais seco).

\section{CPUE}

Através da figura 2 pode-se observar que o maior esforço de pesca foi despendido no verão/97 (1200 $\left.\mathrm{m}^{2} / \mathrm{barco} / \mathrm{dia}\right)$, enquanto que os menores esforços se deram no inverno/97 e na primavera/97 $\left(450 \mathrm{~m}^{2} / \mathrm{barco} /\right.$ dia $)$. Utilizou-se o mesmo esforço de pesca tanto no RA quanto na AC. O esforço de pesca total foi de 7.275 $\mathrm{m}^{2} /$ barco/dia.

A captura foi maior no RA em relação à $\mathrm{AC}$ número de indivíduos e peso dos peixes, sendo esta diferença estatisticamente significativa para ambos os itens $(t=303,8$ e $t=317,2$, respectivamente; $\mathrm{p}<0,05)$. Registraram-se 828 peixes no RA e 278 peixes na $\mathrm{AC}$, e $69,5 \mathrm{~kg}$ no RA e $36,0 \mathrm{~kg}$ na AC (Fig. 3).

A tendência acima se repetiu quanto aos valores de CPUE sobre o número de indivíduos (Fig. 2). Com relação à variação estacional da CPUE observou-se, em geral, superioridade nos valores do RA em relação a AC, com picos máximos no verão/97 e na primavera/96 para a abundância em número de indivíduos $(30,50$ indivíduos $/ \mathrm{m}^{2} /$ barco/dia e 25,11 indivíduos $/ \mathrm{m}^{2} / \mathrm{barco} / \mathrm{dia}$, respectivamente) e mínimos nos inverno/96 e 97 (2,89 indivíduos $/ \mathrm{m}^{2} / \mathrm{barco} / \mathrm{dia}$ e 4,18 indivíduos $/ \mathrm{m}^{2}$ / barco/dia, respectivamente). Observou-se ainda um ciclo sazonal de aumento no verão e queda no inverno. Já a flutuação na $\mathrm{AC}$ foi irregular, com picos máximos 


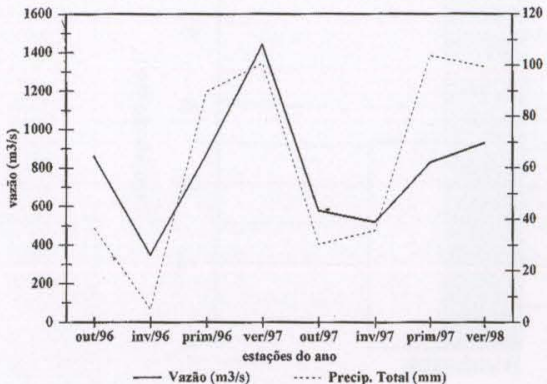

Salinidade

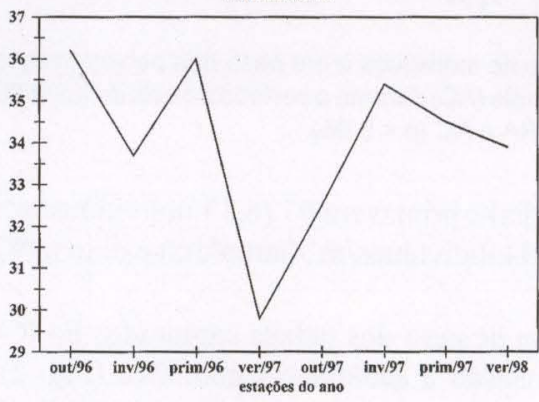

Temperatura da água

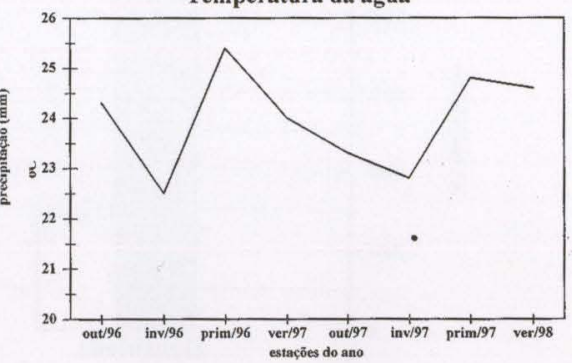

Transparência da água

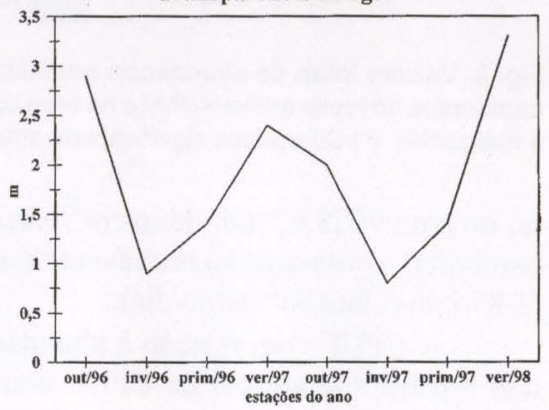

Fig. 1. Variação temporal dos parâmetros físico-químicos analisados ao longo das estações do ano em que o recife artificial foi monitorado. Dados de vazão do rio Paraíba do Sul e de precipitação fornecidos por SouzA et al. (1998).
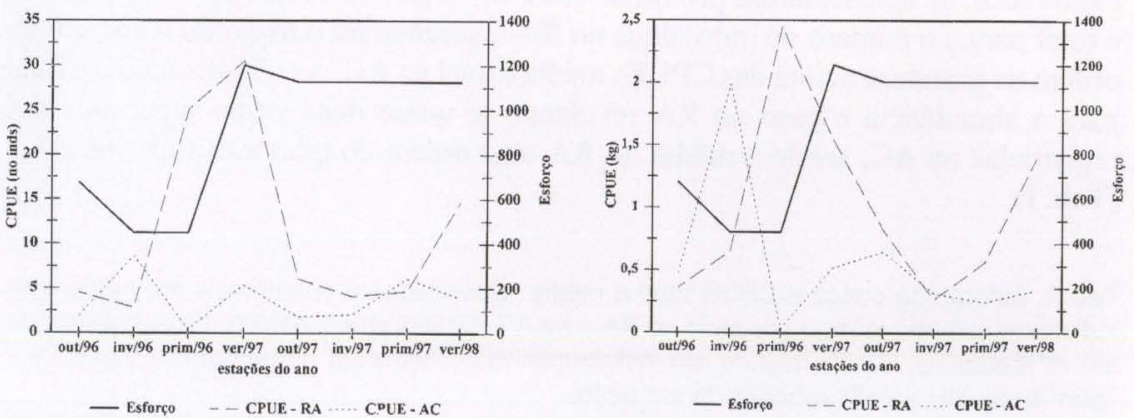

Fig. 2. Variação temporal do esforço de pesca e CPUE relacionados à abundância em número de indivíduos e à abundância em peso no RA e na AC. Observações: esforço de pesca medido em $\mathrm{m}^{2} / \mathrm{barco} / \mathrm{dia}$; CPUE medida em individuo $/ \mathrm{m}^{2} / \mathrm{barco} / \mathrm{dia}$ para a abundância numérica e $\mathrm{kg} / \mathrm{m}^{2} / \mathrm{barco} / \mathrm{dia}$ para a abundância em peso. 


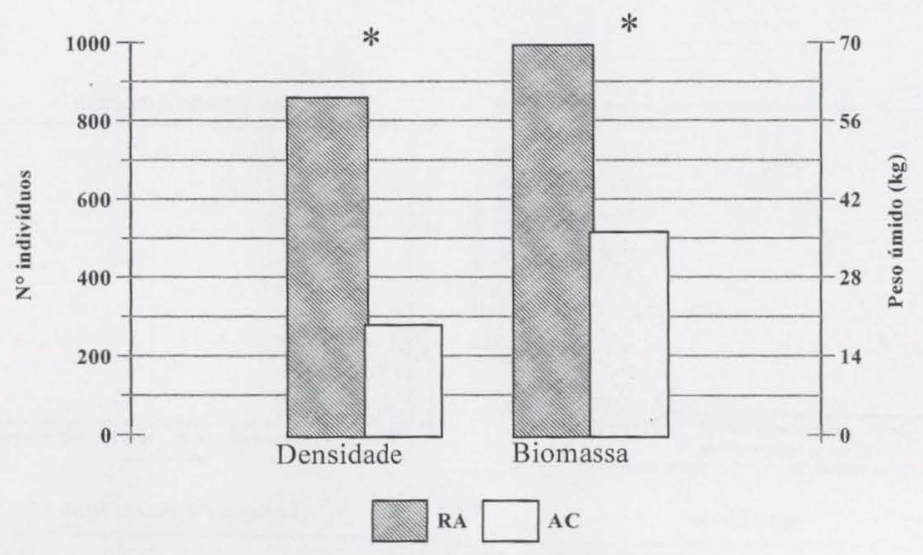

Fig. 3. Valores totais de abundância em número de individuos e em peso dos peixes ósseos capturados no recife artificial (RA) e na área controle (AC) durante o periodo de estudo (abril/96 a março/98). $\left({ }^{*}\right)$ Diferenças significativas entre RA e AC $(p<0,05)$.

no inverno/96 (8,67 indivíduos $/ \mathrm{m}^{2} /$ barco/dia) e primavera/97 (6,13 indivíduos $/ \mathrm{m}^{2} /$ barco/dia), e mínimos na primavera/96 $\left(0,44\right.$ indivíduos $\left./ \mathrm{m}^{2} / \mathrm{barco} / \mathrm{dia}\right)$ e outono/97 (1,87 indivíduos $\left./ \mathrm{m}^{2} / \mathrm{barco} / \mathrm{dia}\right)$.

A CPUE com relação à abundância de peso dos peixes capturados no RA não repetiu a tendência da CPUE com relação à abundância numérica (Fig. 2). Apesar da tendência crescente, os picos máximos ocorreram na primavera/96 $(2,45$ $\left.\mathrm{kg} / \mathrm{m}^{2} / \mathrm{barco} / \mathrm{dia}\right)$ e no verão $/ 97\left(1,55 \mathrm{~kg} / \mathrm{m}^{2} / \mathrm{barco} / \mathrm{dia}\right)$, e os mínimos no inverno/97 $\left(0,27 \mathrm{~kg} / \mathrm{m}^{2} / \mathrm{barco} / \mathrm{dia}\right)$ e outono $/ 96\left(0,36 \mathrm{~kg} / \mathrm{m}^{2} / \mathrm{barco} / \mathrm{dia}\right)$. Já na $\mathrm{AC}$, foram observadas flutuações irregulares, com picos máximos no inverno/96 $\left(1,99 \mathrm{~kg} / \mathrm{m}^{2} /\right.$ barco/dia) e no outono $/ 97\left(0,65 \mathrm{~kg} / \mathrm{m}^{2} / \mathrm{barco} / \mathrm{dia}\right)$, e mínimos nas primavera $/ 96(0,03$ $\mathrm{kg} / \mathrm{m}^{2} /$ barco/dia $)$ e $97\left(0,25 \mathrm{~kg} / \mathrm{m}^{2} /\right.$ barco/dia $)$.

As CPUEs totais e médias, para abundâncias numérica e em peso dos peixes capturados, se apresentaram próximas entre si. Observou-se que as CPUEs média e total para a o número de indivíduos no RA apresentaram o triplo do valor, e uma ordem de grandeza acima das CPUEs média e total na AC. As CPUEs média e total para a abundância e peso no RA revelaram-se quase duas vezes superiores das registradas na $\mathrm{AC}$, sendo a média no $\mathrm{RA}$ uma ordem de grandeza superior à $\mathrm{AC}$ (Tab. I).

Tab. I. Esforço de pesca e CPUE total e média relacionados à abundância em número de individuos e à abundância em peso no RA e na AC. Observaçöes: esforço de pesca medido $\mathrm{em} \mathrm{m}^{2} /$ barco/dia; CPUE medida em individuo $/ \mathrm{m}^{2} /$ barco/dia para a abundância numérica e $\mathrm{kg} / \mathrm{m}^{2} /$ barco/dia para a abundância em peso.

\begin{tabular}{lrr}
\hline \multicolumn{1}{c}{ Pårametros } & Total & Média \\
\hline Esforço & 7275,00 & 909,40 \\
CPUE - RA (número de individuos) & 11,79 & 11,53 \\
CPUE - AC (número de individuos) & 3,82 & 3,82 \\
CPUE - RA $(\mathrm{kg})$ & 0,95 & 1,00 \\
CPUE - AC $(\mathrm{kg})$ & 0,50 & 0,57 \\
\hline
\end{tabular}




\section{Riqueza de espécies}

Nos 23 meses de investigação (abril/96 a março/98), foram capturadas no complexo recifal e na área controle um total de 45 espécies, distribuídas em 41 gêneros e 18 famílias de peixes ósseos (Tab. II). Destas, 30 espécies (67\%) foram comuns ao RA e à AC, enquanto $10(20 \%)$ ocorreram exclusivamente no complexo recifal e $5(13 \%)$ na área-controle, predominando em ambos os sítios representantes das famílias Carangidae e Ariidae (Chloroscombrus crysurus (Linnaeus, 1766) e Sciadeichthys luniscutis (Valenciennes, 1840), respectivamente).

Não foi observado um padrão de sazonalidade com relação à riqueza de espécies, embora valores superiores tenham sido registrados no verão/97 tanto no RA quanto na $\mathrm{AC}$ (Fig. 4). No RA, os maiores valores foram registrados no verão/97 (N $=18$ espécies), seguido pelo outono/97 $(\mathrm{N}=16$ espécies), enquanto os menores valores ocorreram no inverno/96 ( $\mathrm{N}=6$ espécies). $\mathrm{Na} \mathrm{AC}$, os valores mais elevados foram observados no verão/97 e na primavera/97 $(\mathrm{N}=14$ espécies), sendo os menores valores encontrados na primavera/96 ( $N=2$ espécies) e no inverno/97 $(\mathrm{N}=6$ espécies). Foi observada uma riqueza de espécies superior no segundo ano de estudo, em relação ao primeiro ano tanto no $\mathrm{RA}(\mathrm{N}=29$ e $\mathrm{N}=23$ espécies), como na $\mathrm{AC}(\mathrm{N}=24$ e 22 espécies, respectivamente). Diferenças estatisticamente significativas foram observadas entre RA e AC, em relação à riqueza de espécies $(t=324,65 ; \mathrm{p}<0,05)$.
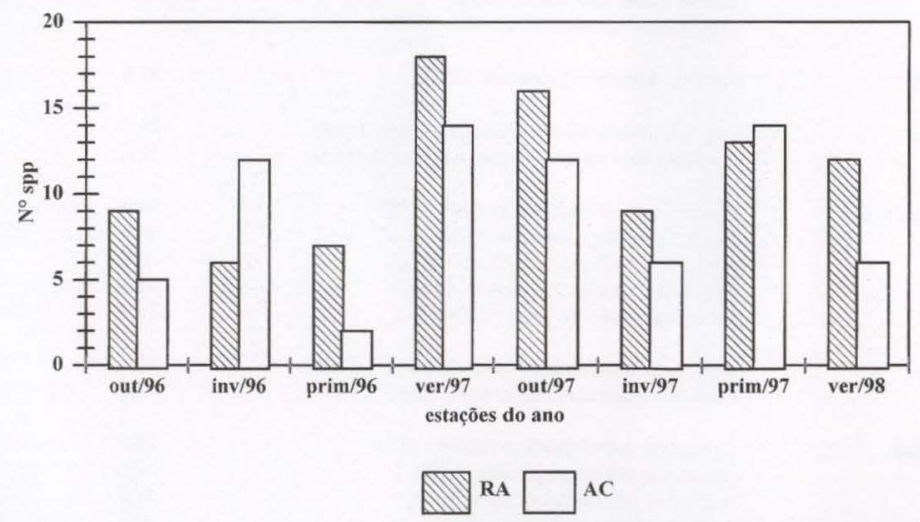

Fig. 4. Variaçăo temporal do número total de espécies capturadas no recife artificial (RA) e na área controle $(\mathrm{AC})$.

\section{Abundância em número de indivíduos}

Um padrão sazonal foi observado na abundância em número dos peixes capturados no RA e na AC (Fig. 5) com valores superiores nos verão/97 e 98 ( $n=366$ e 166 indivíduos, respectivamente), e inferiores no inverno/96 $(\mathrm{n}=13$ indivíduos) e no outono/96 $(\mathrm{n}=31$ indivíduos). Já na $\mathrm{AC}$, o número de indivíduos foi mais elevado na primavera/97 ( $\mathrm{n}=69$ indivíduos) e no verão/97 $(\mathrm{n}=65$ individuos) e inferior na primavera/96 ( $\mathrm{n}=2$ indivíduos) e no outono/96 $(\mathrm{n}=13$ indivíduos). O primeiro ano de estudo superou o segundo em número de indivíduos em ambos os sítios. A AC apresentou-se superior ao recife no inverno/96 e na primavera/97. 
Tab. II. Espécies de peixes capturados no Recife Artificial (RA) e na Área-Controle (AC).

\begin{tabular}{|c|c|c|c|}
\hline Familia & Espécie & RA & $\mathrm{AC}$ \\
\hline Elopidae & Elops saurus (Linnaeus, 1766) & 12,5 & 37,5 \\
\hline \multirow[t]{5}{*}{ Clupeidae } & Odontognathus mucronatus (Lacépède, 1800) & 25,0 & 12,5 \\
\hline & Ophistonema oglinum (LeSeur, 1789) & 75,0 & 37,5 \\
\hline & Pellona harroweri (Fowler, 1917) & 12,5 & 2,5 \\
\hline & Platanicthys platana (Regan, 1917) & 37,5 & 25,0 \\
\hline & Sardinella brasiliensis (Steindachner, 1789) & 37,5 & 7,5 \\
\hline \multirow[t]{3}{*}{ Engraulidae } & Anchoa sp. & - & 2,5 \\
\hline & Anchoviella lepidontostole (Fowler, 1911) & 25,0 & 25,0 \\
\hline & Cetengraulis edentulus (Cuvier, 1828) & - & 2,5 \\
\hline \multirow[t]{4}{*}{ Ariidae } & Bagre bagre (Linnaeus, 1766) & 62,5 & 7,5 \\
\hline & Bagre marinus (Mitchill, 1814) & 12,5 & 2,5 \\
\hline & Genidens genidens (Valenciennes, 1839) & 12,5 & 2,5 \\
\hline & Sciadeicthys iuniscutis (Valenciennes, 1840) & 62,5 & 62,5 \\
\hline Sphyrnidae & Sphyraena guachancho (Cuvier, 1829) & 12,5 & 25,0 \\
\hline Centropomidae & Centropomus paralellus (Poey, 1860) & 12,5 & 12,5 \\
\hline Pomatomidae & Pomatomus saltator (Linnaeus, 1766) & 12,5 & 12,5 \\
\hline \multirow[t]{5}{*}{ Carangidae } & Caranx crysos (Mitchill, 1815) & 62,5 & 37,5 \\
\hline & Caranx latus (Agassiz, 1831) & 37,5 & 25,0 \\
\hline & Chloroscombrus crysurus (Linnaeus, 1766) & 87,5 & 87,5 \\
\hline & Selene vomer (Linnaeus, 1758) & 12,5 & 12,5 \\
\hline & Trachinotus carolinus (Linnaeus, 1766) & - & 12,5 \\
\hline Lutjanidae & Lutjanus synagris (Linnaeus, 1758) & 37,5 & 12,5 \\
\hline \multirow[t]{2}{*}{ Gerreidae } & Diapterus olisthostomus (Goode \& Bean, 1882) & 25,0 & - \\
\hline & Eucinostomus argenteus (Baird \& Girard, 1854) & 12,5 & - \\
\hline \multirow[t]{5}{*}{ Pomadasyidae } & Anisotremus virginicus (Linnaeus, 1758) & 12,5 & - \\
\hline & Conodon nobilis (Linnaeus, 1758) & 37,5 & 37,5 \\
\hline & Haemulon aurolineatum (Cuvier, 1829) & 25,0 & - \\
\hline & Haemulon plumieri (Lacepéde, 1802) & 12,5 & - \\
\hline & Orthopristis ruber (Cuvier, 1830) & 75,0 & 50,0 \\
\hline \multirow[t]{2}{*}{ Sparidae } & Archosargus probatocephalus (Walbaum, 1792) & 25,0 & 12,5 \\
\hline & Callamus pennatula (Guichenot, 1868) & 12,5 & - \\
\hline \multirow[t]{9}{*}{ Sciaenidae } & Cynoscion microlepidotus (Cuvier, 1830) & 25,0 & 37,5 \\
\hline & Cynoscion virescens (Cuvier, 1830) & 12,5 & 12,5 \\
\hline & Isopisthus parvipinnis (Cuvier, 1830) & 12,5 & 25,0 \\
\hline & Larimus breviceps (Cuvier, 1830) & 12,5 & 25,0 \\
\hline & Menticirmus americanus (Linnaeus, 1758) & 12,5 & - \\
\hline & Menticirmus littoralis (Holbrook, 1860) & - & 12,5 \\
\hline & Micropogonias furnieri (Desmarest, 1823) & - & 12,5 \\
\hline & Stellifer rastrifer (Jordan, 1889) & - & 12,5 \\
\hline & Umbrina canosai (Berg, 1895) & 12,5 & 12,5 \\
\hline Mullidae & Pseudupeneus maculatus (Bloch, 1793) & 25,0 & - \\
\hline Ephippidae & Chaetodipterus faber (Broussonet, 1782) & 37,5 & - \\
\hline Polynemidae & Polydactylus oligodon (Günther, 1860) & 37,5 & - \\
\hline Trichiuridae & Trichiurus lepturus (Linnaeus, 1758) & 25,0 & 37,5 \\
\hline Scombridae & Scomberomorus maculatus (Mitchill, 1815) & 12,5 & 25,0 \\
\hline Total de espécies & & 45 & 39 \\
\hline
\end{tabular}



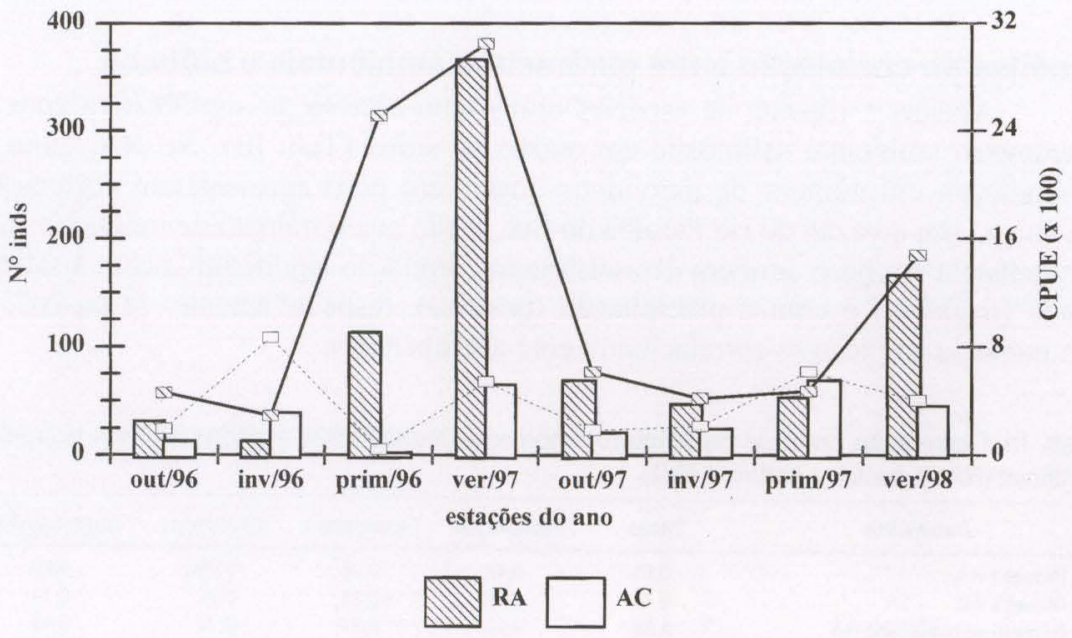

Fig. 5. Variação temporal da abundância em número de indivíduos no recife artificial (RA) e na área controle (AC). Linhas indicam a CPUE correspondente de cada período, para RA e $A C$.
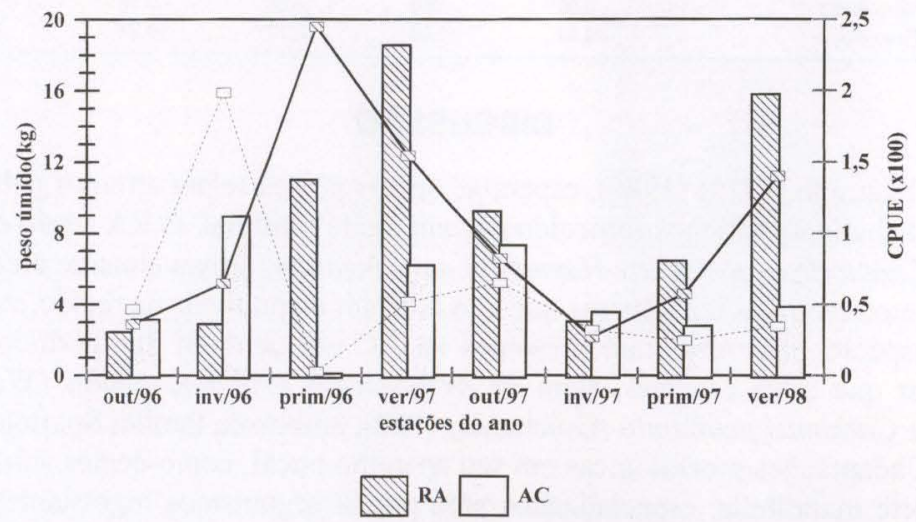

Fig. 6. Variação temporal da abundância de peso no recife artificial (RA) e na área controle (AC). Linhas indicam a CPUE correspondente de cada periodo, para RA e AC.

\section{Abundância em peso}

Uma sazonalidade foi observada também com relação à abundância em peso dos peixes capturados no RA e na AC (Fig. 6), sendo que os maiores valores observados no RA ocorreram no verão/97 $(8,55 \mathrm{~kg})$ e no verão/98 $(15,78 \mathrm{~kg})$, enquanto os menores foram registrados no inverno/96 $(2,44 \mathrm{~kg})$ e no outono/96 $(2,91 \mathrm{~kg})$. Já no controle, os valores de peso foram maiores no inverno/96 $(8,34$ $\mathrm{kg})$ e no outono $/ 97(7,33 \mathrm{~kg})$ e também menores nas primavera $/ 96(0,12 \mathrm{~kg})$ e 97 $(2,79 \mathrm{~kg})$. Da mesma forma, o segundo ano superou o primeiro em ambos os sítios. 


\section{Análise de correlação entre parâmetros ambientais e bióticos}

Apenas a riqueza de espécies apresentou correlação significativa com o parâmetro ambiental salinidade em ambos os sítios (Tab. III). No RA, tanto a abundância em número de indivíduos quanto em peso apresentaram correlação positiva com a vazão do rio Paraíba do Sul, sendo que o número de indivíduos e a abundância em peso também demonstraram correlação significativa com a salinidade (negativa) e com a precipitação (positiva), respectivamente. Já na $\mathrm{AC}$, a abundância em peso se correlacionou com a temperatura.

Tab. III. Correlação entre os parâmetros ambientais monitorados e dados bióticos no recife artificial $(R A)$ e na área controle $(A C)$.

\begin{tabular}{lccccr}
\hline \multicolumn{1}{c}{ Parâmetros } & Vazão & Precipitaçắo & Temperatura & Salinidade & Transparència \\
\hline Riqueza RA & 0,61 & 0,43 & 0,08 & $-0,79^{*}$ & 0,42 \\
Riqueza AC & 0,10 & 0,01 & $-0,36$ & $-0,74^{*}$ & $-0,14$ \\
Número de individuos RA & $0,88^{*}$ & 0,63 & 0,27 & $-0,75^{*}$ & 0,43 \\
Número de individuos AC & 0,40 & 0,46 & $-0,02$ & $-0,62$ & 0,08 \\
PesoRA & $0,78^{*}$ & $0,73^{*}$ & 0,43 & $-0,66$ & 0,52 \\
Peso AC & $-0,27$ & $-0,55$ & $-0,79^{*}$ & $-0,64$ & $-0,08$ \\
CPUE (número de individuos RA) & $0,81^{*}$ & 0,66 & 0,51 & $-0,45$ & 0,29 \\
CPUE (número de indivíduos AC) & $-0,08$ & $-0,07$ & $-0,37$ & $-0,43$ & $-0,20$ \\
CPUE (Peso RA) & 0,50 & 058 & 0,62 & $-0,16$ & 0,17 \\
CPUE (Peso AC) & $-0,50$ & $-0,68$ & $-0,72 *$ & $-0,24$ & $-0,30$ \\
\hline
\end{tabular}

\section{DISCUSSÃO}

Segundo D'ITRI (1986), espera-se que os peixes sejam atraídos pela maior oferta de habitats e nichos fornecido por um recife artificial. O RA atraiu espécies como Chaetodipterus faber e Haemulon aurolineatum, provavelmente oferecendo abrigo e/ou recursos alimentares que não estavam disponíveis na região, visto que essas espécies não estiveram presentes na $\mathrm{AC}$ em nenhum dos períodos. Vale salientar que estas espécies, além de Archosargus probatocephalus (Walbaum, 1792) e Callamus pennatula (Guichenot, 1868), ambos da família Sparidae, apresentam adaptações morfológicas em seu aparelho bucal, como dentes achatados e uma forte mandibula, especializados para predar organismos incrustantes. Além dessas, outras espécies, como Pseudupeneus maculatus (Bloch, 1793), Diapterus olisthostomus (Goode \& Bean, 1882), Eucinostomus argentum (Baird \& Girard, 1854) e Polydactylus oligodon (Günter, 1860), contribuíram para um incremento de espécies no RA, especialmente nos meses de primavera e verão. As espécies que se apresentaram exclusivamente na $\mathrm{AC}$ pertenciam às famílias Engraulidae e Sciaenidae.

Das espécies que ARAÚJO (1996) caracterizou como típicas do estuário do rio Paraíba do Sul, nove foram encontradas também na região de Manguinhos, porém em proporções inversas. Tal fato levanta a hipótese da região ser influenciada por outros fatores além do referido rio.

Peixes que predam sobre substrato consolidado capturados neste estudo eram raros ou desconhecidos dos pescadores. Estudos realizados em zona de arrebentação na costa brasileira (MONTEIRO-NETO et al. 1990) e no golfo do México (NAUGHTON 
\& SALOMAN 1978; FOX \& MOCK 1968) registraram algumas destas espécies bentívoras. Tal ambiente é tido como área de berçário, tendo assim importante função no ambiente costeiro, sendo influenciados diretamente pelos estuários. Uma hipótese seria que estes peixes viriam da zona de arrebentação em função da proximidade relativa do recife com a praia (cerca de cinco milhas). Além disso, estudos em zona de arrebentação na costa norte do estado do Rio de Janeiro vêm sendo realizados no presente momento para investigar a influência dos estuários na composição ictiofaunística dessa região. Uma vez que tal ambiente é importante para a entrada de peixes jovens, os estudos poderão indicar se existe alguma relação em termos de composição e abundância de peixes entre este ambiente e o recife artificial.

No Chile, JARA \& CÉSPEDES (1994), e no Mar Mediterrâneo, MORENO et al. (1994) e SANTOS \& MONTEIRO (1997), verificaram, também em experimentos com estruturas artificiais de grande porte, uma maior abundância em número de indivíduos e em peso de peixes no recife artificial do que em áreas-controle. No presente estudo, o RA revelou maior poder atrator nos meses de verão, evidenciado pelos altos valores de abundância nesse período decorrente do incremento populacional de Chloroscombrus crysurus na região, possivelmente obedecendo a um ciclo migratório. ARAÚJO et al. (1998) relacionaram esta espécie como freqüentadora de zonas estuarinas, com densidades superiores em águas mais turvas. No inverno/96, o RA pode ter tido seu poder atrator diminuído em função do aumento da turbidez na região, favorecendo espécies mais aptas às condições apresentadas.

Poucos estudos com recifes artificiais utilizam valores de peso como atributo numérico devido à metodologia de amostragem aplicada, que na maioria das vezes é o censo visual por mergulho autônomo. Ainda, diferentes metodologias de amostragem podem evidenciar diferentes agrupamentos de peixes (SANTOS \& MONTEIRO 1997), devido à seletividade das mesmas. Neste estudo, foi observado que, além do número de indivíduos, o peso apresentou-se quase duas vezes superior no RA. Os padrões de incrustação podem ter influenciado as taxas de colonização de peixes no $\mathrm{RA}$, visto que os organismos que pertencem a níveis tróficos mais baixos da cadeia alimentar, atraem herbívoros e grazers (SAMMARCO et al. 1987), assim como acontece em recifes naturais. O complexo recifal foi instalado em março/96, sendo a incrustação gradualmente incrementada com o passar do tempo. Tal fato poderia explicar os baixos valores de peso dos peixes capturados nos dois primeiros períodos no RA, mas que, com o passar do tempo, apresentou uma tendência a um incremento e, inclusive, superioridade em relação aos valores de biomassa na AC.

Os valores de peso mais elevados nos verões de 1997 e 1998, decorrentes do incremento da população de $C$. crysurus, evidenciam sua preferência pelo RA. Entretanto, os indivíduos desta espécie encontravam-se notadamente em época reprodutiva. É conhecido que fềmeas armazenam energia em forma de gordura na época de reprodução, podendo mascarar o aproveitamento na incorporação dos nutrientes ao peso do peixe nesta época (VAZZOLER 1996).

Ao longo dos meses de amostragem, as maiores CPUEs foram registradas no RA durante o verão/97, primavera/96 e verão/98, correspondendo ao período de concentração da população de C. crysurus e à presença de espécies exclusivas no RA, como $C$. faber e $H$. aurolineatum. Os picos de CPUE com relação ao número de 
indivíduos ocorreram no inverno/96, verão/97 e primavera/97, sob influência do aumento das populações de Ariidae (Bagre bagre (Linneus, 1766) e Sciadeichthys luniscutis), C. crysurus, e Clupeidae [Odontognathus mucronatus (Lacépède, 1800), Ophistonema oglinum (Lesueus, 1789), Platanichthys platana (Regan, 1917) e Sardinella brasiliensis (Steindachner, 1789)], respectivamente. Já os picos de CPUE com relação ao peso dos peixes capturados ocorreram no inverno/96, verão/97 e outono/97, sob influência do aumento das populações de Ariidae (B. bagre e S. luniscutis), C. crysurus e da presença de Elops saurus (Linnaeus, 1766), respectivamente.

Os picos máximos de CPUE, tanto para a densidade quanto para a biomassa dos peixes capturados no RA não coincidiram com os picos máximos na $\mathrm{AC}$, o que pode significar que o RA possui um período ótimo de desenvolvimento de sua comunidade diferente da AC. Projetando para médio/longo prazo, a atividade pesqueira na região poderia ser incrementada. CONCEIC̣Ão \& MoNTEIRO-NETO (1998) afirmaram que se deve reconhecer o papel dos recifes artificiais na redução das taxas de mortalidade natural, preservando uma biomassa que seria perdida dentro dos processos de competição e predação na comunidade marinha. São necessários também mais estudos para se determinar que tipo de impacto(s) o aumento nas dimensões do RA, para utilização em uma escala comercial, poderia causar.

O número de peixes capturados no RA pode ter sido influenciada positivamente pela vazão, mas de maneira indireta. Na medida em que são disponibilizados nutrientes para serem aproveitados pelas comunidades incrustantes do RA e outros detritívoros, a base da cadeia alimentar estaria sendo reforçada, atraindo cada vez mais peixes. São necessários estudos mais específicos sobre os padrões de circulação e as variáveis ambientais que regem esta região para relacioná-los com a comunidade de peixes ósseos.

O peso de peixes no RA apresentou uma correlação positiva com a vazão do rio Paraíba do Sul e a precipitação, o que reflete mais uma vez suas influências na base da cadeia trófica e nos peixes plancto-detritivoros. A influência da precipitação está na sua relação direta com a vazão.

A correlação negativa entre a riqueza de espécies e a salinidade, em ambos os sítios, pode refletir que parte das espécies capturadas seja típica de ambientes estuarinos. É o caso de famílias como Elopidae, Clupeidae, Engraulidae e Ariidae, entre outras (FIGUEIREDO \& MENEZES 1978).

Vale ressaltar a correlação significativa dos valores de riqueza de espécies, número de indivíduos e peso dos peixes capturados com as variáveis ambientais salinidade, vazão do rio Paraíba do Sul e precipitação. Tais resultados indicam claramente $o$ efeito da precipitação na salinidade, evidenciando a importância do regime de chuvas na distribuição de peixes ósseos, sob ação direta do rio Paraíba do Sul na região.

AGRADECIMENTOS. Agradecemos a FENORTE, FAPERJ e CNPq; ao Curso de Bacharelado em Ciências Biológicas da Universidade Estadual do Norte Fluminense; ao apoio de MsC. Flávio Antônio Costa Gomes, do MsC. Eduardo Ayres Godoy de Souza; e do apoio técnico do Sr. Antônio Carlos de Oliveira Peçanha.

Revta bras. Zool. 18 (3): 779 - 792, 2001 


\section{REFERÊNCIA BIBLIOGRÁFICAS}

ArAújo, F.G. 1996. Composição e estrutura da comunidade de peixes do médio e baixo rio Paraíba do Sul, RJ. Rev. Brasil. Biol. 56 (1): 111-126.

Araúso, F.G.; A.G. Cruz-Filho; M.C.C. AzevÊdo \& A.C.A. SAntos. 1998. Estrutura da comunidade de peixes demersais da baía de Sepetiba, RJ. Rev. Brasil. Biol. 58 (3): 417-430.

BoHnSACK, J.A.; A.M. ECKLUND \& A.M. SzMANT. 1997. Artificial Reef Research: Is There More Than the Attraction-Production Issue? Fisheries 22 (4): 14-12.

Bombace, G.; G. Fabi; L. Fiorentini \& S. Speranza. 1994. Analysis of the efficacy of artificial reefs located in five different areas of the Adriatic Sea. Bull. Mar. Sci. 55 (2-3): 559-580.

Conceiçĩo, R.N.L. \& C. Monteiro-Neto. 1998. Recifes artificiais marinhos. Biotec. Ci. \& Desenvol., São Paulo, 6: 14-17.

D'ITRI, F.M. 1986. Artificial Reefs: Marine and Freshwater Applications. Chelsea, Lewis Publ. Inc., $589 \mathrm{p}$.

Figueiredo, J.L. \& N.A. Menezes. 1978. Manual de peixes marinhos do sudeste do Brasil. II. Teleostei (1). São Paulo, Museu de Zoologia, Univ. São Paulo, 110p.

1980. Manual de peixes marinhos do sudeste do Brasil. III. Teleostei (2). São Paulo, Museu de Zoologia, Univ. de São Paulo, 90p.

Fox, L.S. \& W.R. Mock JR. 1968. Seasonal Occurrence of Fishes in Two Shore Habitats in Barataria Bay, Luisiana. Louis. Acad. Sci. 31: 43-53.

Grove, R.S.; M. NaKamura; H. KaKimoto \& C.J. Sonu. 1994. Aquatic habitat technology innovation in Japan. Bull. Mar. Sci. 55 (2-3): 276-294.

J^COBI, C.M. 1994. Sucessão em Comunidades Experimentais Aquáticas. An. III Simp. Ecos. Costa Brasileira, Serra Negra, 3: 246-255.

JARA, F. \& R. CÉSPEDES. 1994. An experimental evaluation of habitat enhancement on homogeneous marine bottoms in southern Chile. Bull. Mar. Sci. 55 (2-3): 295-307.

Lindquist, D.G.; L.B. Cahoon; I.E. Clıvijo; M.H. Posey; S.K. Bolden; L.A. Pike; S.W. BurK \& P.A. CARDullo. 1994. Reef fish stomach contents and prey abundance on reef and sand substrata associated with adjacent artificial and natural reefs in Onslow Bay, North Carolina. Bull. Mar. Sci. 55 (2-3): 308-318.

Menezes, N.A. \& J.L. Figueiredo. 1980. Manual de peixes marinhos do sudeste do Brasil. IV. Teleostei (3). São Paulo, Museu de Zoologia, Univ. São Paulo, 96p.

- . 1985. Manual de peixes marinhos do sudeste do Brasil. V. Teleostei (4). São Paulo, Museu de Zoologia, Univ. de São Paulo, 105p.

Monteiro-Neto, C.; C. Blacher; A.A.S. Laurent; F.N. Snizek; M.B. Canozzi \& L.L.C. De A. TABAJARA. 1990. Estrutura da Comunidade de Peixes em Águas Rasas na Região de Laguna, Santa Catarina, Brasil. Atlântica, Rio Grande, 12 (3): 53-69.

Moreno, I.; I. Roca; O. Reñones; J. Coll \& M. SalamanCa. 1994. Artificial reef program in Balearic waters (Western Mediterranean). Bull. Mar. Sci. 55 (2-3): 667-671.

MotTET, M.G. 1986. Enhancement of the Marin Environment for Fisheries, p. 13-112. In: F.M. D'ITRI (Ed.). Artificial Reefs: Marine and Freshwater Applications. Chelsea, Lewis Publ. Inc., 589p.

Naughton, S.P. \& C.H. Saloman. 1978. Fishes of the Nearshore Zone of St. Andrew Bay, Florida, and Adjacent Coast. Nor. Gulf Sci. 2 (1): 43-55.

Pearce, J.B. \& S. Chang. 1982. The efficacy of various materials in artificial reef construction. Int.

Counc. Expl. Sea. 64: 1-11.

Risk, M.J. 1981. Artificial reefs in Discovery Bay, Jamaica. Atoll. Res. Bull. 225: 91-100.

RYU, H.Y. 1995. Artificial reef ecology. Kyoto, $9^{\text {th }}$ Marine Ranch Training Course in Koshi Univ., $13 \mathrm{p}$.

SAMmaRCO, P.W.; M.J. Risk \& C. ROSE. 1987. Effects of grazing and damselfish territoriality on internal bioerosion of dead corals: indirect effects. Jour. Exp. Mar Biol. Ecol. 112: 185-199.

Santos, M.N. \& C.C. Monteiro. 1997. The Olhao Artificial Reef System (South Portugal) - Fish Assemblages and Fishing Yield. Fish. Res. 30 (1-2): 33-41. 
Seaman JR., W.; R.M. Buckley \& J.J. Polovina. 1989. Advances in Knowledge and Priorities for Research and Mangement related to Artificial Aquatic Habitats. Bull. Mar. Sci. 44 (2): 527-532. SoKAL, R.P. \& F.J. RoHLF. 1981. Biometry: The Principles and Practice of Statistics in Biological Research. San Francisco, W.H. Freudman Co., $2^{\text {nd }}$ ed., 776p.

SouzA, E.G.A.; C.F. SilvA; E.A. PEReira \& I.R. ZALmon. 1998. A influência de parâmetros ambientais na distribuição temporal dos peixes capturados em recife artificial (S. Francisco do Itabapoana, RJ). An. IV Simp. Ecos. Brasil., Águas de Lindóia, 1: 325-332.

StonE, R.B. 1986. History of Artificial Reef Use in the United States, p. 1-12. In: F.M. D'ITRI (Ed.) Artificial Reefs: Marine and Freshwater Applications. Chelsea, Lewis Publ. Inc., USA, 589p.

VALENTIN, J.L. \& W.M. MONTEIRO-RiBAS. 1993. Zooplankton community structure on the east-southeast Brazilian continental shelf (18-23ㅇ latitude). Cont. Shelf Res. 13 (4): 407-424.

V^zZOLER, A.E.A.M. 1996. Biologia da reprodução de peixes teleósteos: teoria e prática. Maringá, Ed. Univ. Estadual Maringá, 169p.

Zalmon, I.R.; B.A.P. GamA; \& A. LetA. 1993. Fouling Community at Guanabara Bay, Brazil: A Directional Process of a Variable Temporal Progression? Oebalia 19 (Suppl.): 217-222.

ZAR, J.H. 1999. Biostatistical Analysis. New Jersey, Prentice-Hall, $4^{\text {th }}$ ed., 663p.

Recebido em 30.III.2000; aceito em 24.VII.2001 